\title{
KÖTHE'S EXAMPLE OF AN INCOMPLETE LB-SPACE
}

\author{
J. KUCERA AND K. MCKENNON
}

\begin{abstract}
The inductive limit of reflexive Banach spaces is fast complete. But the inductive limit of reflexive Fréchet spaces may not be fast complete. Also, an example of a complete, reflexive, nuclear space with a quotient space which is not fast complete is given.
\end{abstract}

In $[\mathbf{1}, \S 31.6]$ Köthe presents a sequence of Banach spaces whose inductive limit is not quasi-complete. It is shown in [4 and 5] that inductive limit of a sequence of reflexive Banach spaces is complete. Here we construct a sequence of quite natural nuclear Fréchet (hence reflexive) spaces $E_{n}$ whose ind $\lim E_{n}$ is not fast complete, and therefore it is neither sequentially nor quasi-complete.

As in $[\mathbf{3}]$, for an absolutely convex set $A$ in a locally convex space $X$, we denote by $X_{A}$ the linear hull of $A$ equipped with the topology generated by $\{\lambda A ; \lambda>0\}$. If $X_{A}$ is a Banach space, we call $A$ a Banach disk. The space $X$ is fast complete if each set bounded in $X$ is contained in a bounded Banach disk.

Both sequential and quasi-completeness are defined in $[\mathbf{1}]$ and the latter implies the former $[\mathbf{1}, \S 18.4]$. Every sequentially complete locally convex space $X$ is fast complete.

To prove it, take a set $A$ bounded in $X$ and denote by $B$ the absolutely convex closed hull of $A$ in $X$. It is sufficient to show that $B$ is a Banach disk. Let $\left\{x_{n}\right\}$ be a Cauchy sequence in $X_{B}$. Since $B$ is bounded in $X$, the identity map $X_{B} \rightarrow X$ is continuous, and $\left\{x_{n}\right\}$ is a Cauchy sequence in $X$. Let $x_{0} \in X$ be its limit (in the topology of $X$ ). Further, $\left\{x_{n}\right\}$ is bounded in $X_{B}$ and therefore it is contained in $\lambda B$ for some $\lambda>0$. But $\lambda B$ is closed in $X$, hence $x_{0} \in \lambda B \subset X_{B}$, which implies the convergence of $\left\{x_{n}\right\}$ to $x_{0}$ in the topology of $X_{B}$.

Let $E_{1} \subset E_{2} \subset \cdots$ be Fréchet spaces with continuous id: $E_{n} \rightarrow E_{n+1}$ for each $n \in N$, and $E=$ ind $\lim E_{n}$. It is proved in $[1, \S 19.5(5)]$ that if $E$ is quasi-complete then each set bounded in $E$ is contained and bounded in some $E_{n}$. This result cannot be reversed. But Qiu Jing Huei showed in [5] that $E$ is fast complete iff each set bounded in $E$ is contained and bounded in some $E_{n}$.

Let $D_{n}=(0, \infty) \backslash\{1,2, \ldots, n\}, n \in N$, and let $E_{n}$ be the space $\mathfrak{C}^{\infty}\left(D_{n}\right)$ with topology generated by the seminorms

$$
\|f\|_{n, m}=m \cdot \sup \left\{\left|f^{(i)}(x)\right| ; x \in[0, m], \begin{array}{r}
|x-j| \geq 1 /(m+1), \\
i=0,1, \ldots, m, j=0,1, \ldots, n\},
\end{array}\right.
$$

$m=1,2, \ldots$ Then each $E_{n}$ is a Fréchet space, $E_{1} \subset E_{2} \subset \cdots$, all identity maps $E_{n} \rightarrow E_{n+1}$ are continuous, and $E=$ ind $\lim E_{n}$ is Hausdorff.

Received by the editors February 15, 1983 and, in revised form, January 30, 1984.

1980 Mathematics Subject Classification. Primary 46A12; Secondary 46A14, 46A45, 46E10.

Key words and phrases. LB-space, LF-space, completeness, fast completeness. 
Each set $D_{n}$ is open, hence each $E_{n}$ is the inductive limit of a sequence of L. Schwartz spaces $\mathfrak{D}_{K}$, where $K \subset D_{n}$ is compact. The spaces $\mathfrak{D}_{K}$ are nuclear $[\mathbf{2}$, Chapter 3. §8], and their countable inductive limits $E_{n}$, as well as $E=\operatorname{ind} \lim E_{n}$, are nuclear too. Since nuclear Fréchet spaces are Montel, each $E_{n}$ is Montel and, as such. reflexive.

Let $f_{n}(x)=c_{n}(x-n)^{n-1 / 2} \exp (n-x), x \in(0, \infty), n \in N$, where $c_{n}>0$ is chosen so that $\sup \left\{\left|f_{n}^{(i)}(x)\right| ; x \in(0, \infty), i=0,1, \ldots, n-1\right\} \leq 1 / n$. Since $f_{n}$ is infinitely differentiable everywhere on $(0, \infty)$ but $x=n$, we have $f_{n} \in E_{n} \backslash E_{n-1}, E_{0}=$ $\{0\}, n \in N$. Put $B=\left\{f_{n} ; n \in N\right\}$. Then $B \not \subset E_{n}$ for every $n \in N$ and a fortiori $B$ is not bounded in $E_{n}$. Hence to prove that $E$ is not fast complete, it is sufficient to show that $B$ is bounded in $E$.

Take 0-neighborhoods $U, V$ in $E$ such that $U+U \subset V$. There exists $m \in N$ such that the \|\|$_{1, m}$-unit ball $B_{1, m} \subset U$. Fix this $m$ and take $n>m$. There exist $k>n$ and $\lambda>0$ such that $\lambda B_{n, k} \subset U$. Let $g_{n} \in \mathfrak{C}^{\infty}(0, \infty)$ be such that $g_{n}(x)=f_{n}(x)$ for $x \in(0, \infty) \backslash[n-1 / k, n+1 / k]$. Then $g_{n} \in E_{1} \subset E_{n},\left\|f_{n}-g_{n}\right\|_{n, k}=0$, and

$$
\begin{aligned}
\left\|g_{n}\right\|_{1, m} & =\left\|f_{n}\right\|_{1, m} \\
& \leq m \cdot \sup \left\{\left|f_{n}^{(i)}(x)\right| ; x \in(0, \infty), i=0,1, \ldots, n-1\right\} \\
& \leq m / n<1 .
\end{aligned}
$$

Hence $g_{n} \in B_{1, m}$ and $f_{n}=\left(f_{n}-g_{n}\right)+g_{n} \in U+U \subset V$. Now, for $\alpha>1$ such that $f_{k} \in \alpha V, k=1,2, \ldots, m$. we have $B \subset \alpha V$ and $B$ is bounded in $E$.

Since all $E_{n}$ are complete, reflexive, and nuclear, their direct sum $\bigoplus E_{n}$ is complete, reflexive, and nuclear too. The mapping $T:\left(x_{1}, x_{2}, \ldots\right) \mapsto \sum x_{n}: \bigoplus E_{n} \rightarrow$ ind $\lim E_{n}$ induces an isomorphism $\left(\bigoplus E_{n}\right) / \operatorname{ker} T \rightarrow$ ind $\lim E_{n}[\mathbf{1}, \S 19.5]$. Thus $\bigoplus E_{n}$ is an example of a complete, reflexive, nuclear space with a fast incomplete quotient space.

\section{REFERENCES}

1. G. Köthe, Topological vector spaces. I, Springer, Berlin and New York, 1969.

2. H. Schaefer, Topological vector spaces, 3rd ed., Springer, Berlin and New York, 1971.

3. M. de Wilde, Closed graph theorems and webbed spaces, Pitman, London, 1978.

4. J. Kucera and K. McKennon, Dieudonné-Schwartz theorem on bounded sets in inductive limits, Proc. Amer. Math. Soc. 78 (1980), 366-368.

5. Qiu Jing Huei, Some results on bounded sets in inductive limits (to appear).

Department of Pure and Applied Mathematics, Washington State UniverSity, Pullman, Washington 99164-2930 